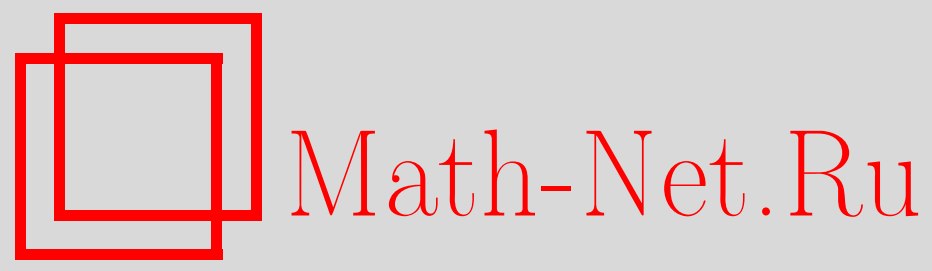

Б. И. Селиванов, О вычислении допредельных распределений разделимых статистик полиномиальной схемы, Дискрет. матем., 2006, том 18, выпуск 3, 85-94

DOI: https://doi.org/10.4213/dm61

Использование Общероссийского математического портала Math-Net.Ru подразумевает, что вы прочитали и согласны с пользовательским соглашением http://www.mathnet.ru/rus/agreement

Параметры загрузки:

IP: 54.209 .52 .79

26 апреля 2023 г., 17:28:51 


\title{
О вычислении допредельных распределений разделимых статистик полиномиальной схемы
}

\author{
() 2006 г. Б. И. Селиванов
}

\begin{abstract}
Представлено несколько рекуррентных процедур вычисления допредельных (точных) распределений разделимых статистик полиномиальной схемы. Для получения рекуррентных соотношений используются производящие функции рассматриваемых статистик. В самом общем случае предлагаемый способ вычисления сводится к алгоритму А. М. Зубкова (1996), изложенному на языке цепей Маркова.

Работа выполнена при поддержке программой Президента Российской Федерации поддержки ведущих научных школ, проект НШ 1758.2003.1.
\end{abstract}

\section{1. Введение}

Рассмотрим последовательность независимых одинаково распределенных случайных величин

$$
X_{1}, \ldots, X_{n}, \ldots,
$$

принимающих значения $1, \ldots, N$. В дальнейшем нам будет удобнее называть (1) последовательностью (однородных) независимых испытаний с возможными исходами $1, \ldots, N$ или просто выборкой. Частоту исхода $i$ в испытаниях (1) будем обозначать $v_{i}=v_{i}(n)$, $i=1, \ldots, N$, ясно, что $\sum_{i=1}^{N} v_{i}=n$.

Каждое из испытаний (1) проводится с использованием одной и той же полиномиальной схемы. Если эта схема такова, что вероятность появления исхода $i$ равна $q_{i}$,

$$
0 \leqslant q_{i} \leqslant 1, \quad i=1, \ldots, N, \quad \sum_{i=1}^{N} q_{i}=1,
$$

то говорим, что выполняется (простая) гипотеза $H_{q}$, где $q=\left(q_{1}, \ldots, q_{N}\right)$. Из множества гипотез $H_{q}$ выделим и зафиксируем гипотезу $H=H_{p}$, при выполнении которой вероятности исходов равны $p_{1}, \ldots, p_{N}$, соответственно, и

$$
0<p_{i}<1, \quad i=1, \ldots, N, \quad \sum_{i=1}^{N} p_{i}=1
$$

гипотезу $H$ назовем нулевой. Нулевую гипотезу, в соответствии с которой реализуется равновероятная полиномиальная схема, то есть схема с $p_{1}=\ldots=p_{N}=1 / N$, обозначим 
$H_{0}$. Все гипотезы $H_{q}$, для которых $q=\left(q_{1}, \ldots, q_{N}\right) \neq\left(p_{1}, \ldots, p_{N}\right)=p$, будем называть альтернативами нулевой гипотезе (простыми конкурирующими гипотезами).

Пусть даны функции $f_{1}(x), \ldots, f_{N}(x)$, определенные для целых $x \geqslant 0$ и принимающие действительные значения. Случайная величина

$$
L(n, N)=\sum_{i=1}^{N} f_{i}\left(v_{i}\right), \quad n=1,2, \ldots,
$$

называется разделимой статистикой полиномиальной схемы. Если для всех целых $x \geqslant 0$ выполняются равенства $f_{1}(x)=\ldots=f_{N}(x)$, то разделимая статистика называется симметричной. Класс разделимых статистик включает в себя (при соответствующей нормировке и центрировке) статистики, чаще всего используемые при статистическом анализе случайных последовательностей. К этому классу, в частности, принадлежит известная статистика хи-квадрат К. Пирсона (см., например, [1], раздел 30.1).

В настоящее время задача о предельных распределениях разделимых статистик при $n \rightarrow \infty$ и $N$, фиксированном или стремящемся к бесконечности, изучена достаточно хорошо. На практике при построении и расчете статистических критериев нередко возникает вопрос о возможности использования предельных распределений разделимых статистик в допредельном случае для конечных значений $n$ и $N$. Однозначного ответа на этот вопрос нет. В статистической литературе высказываются различные соображения о степени аппроксимации точного распределения статистики хи-квадрат, упомянутой выше, предельным хи-квадрат распределением, см., например, высказывания на эту тему Г. Крамера ([1], с. 457), Б. Л. ван дер Вардена ([2], с. 275), Г. И. Ивченко и Ю. И. Медведева ([3], c. 110-111).

Прямой переборный метод нахождения точных распределений статистик (3) с использованием полиномиального распределения вектора частот $\left(v_{1}, \ldots, v_{N}\right)$ в вычислительном отношении достаточно сложен. В настоящей работе обсуждаются способы вычисления распределений разделимых статистик полиномиальной схемы, при которых применяются рекуррентные процедуры. В их основе лежит представление для производящих функций этих статистик.

В разделе 2 рассматривается одно частное множество разделимых статистик, а именно, статистики вида

$$
\zeta_{n}^{(m)}=\sum_{i=1}^{N}\left(\begin{array}{c}
v_{i}(n) \\
m
\end{array}\right), \quad m=2,3, \ldots
$$

Заметим, что при $m=2$ выполняется соотношение

$$
\chi_{n}^{2}=\frac{2 N}{n} \zeta_{n}^{(2)}+N-n
$$

где

$$
\chi_{n}^{2}=\frac{N}{n} \sum_{i=1}^{N}\left(v_{i}(n)-n / N\right)^{2}=\frac{N}{n} \sum_{i=1}^{N} v_{i}^{2}(n)-n .
$$

Статистика (7) есть статистика хи-квадрат, применяемая для проверки согласия с гипотезой $H_{0}$.

Вычислением допредельного или точного распределения статистики $\chi_{n}^{2}$ в предположении справедливости гипотезы $H_{0}$ занимались многие авторы. В частности, в статьях [4] и 
[5] указаны точные значения “хвостовых" областей распределения $\chi_{n}^{2}$ для ряда значений $n$ и $N$, найденные с использованием двумерного дискретного преобразования Фурье. В [4] и [5] содержатся также дополнительные библиографические сведения о работах в этой области.

В разделе 2 предлагается подход к вычислению допредельных распределений $\chi_{n}^{2}$ и статистик вида (5), при котором используются результаты автора (см. [6]), опубликованные в 1972 г. и представляющие еще, по мнению автора, определенный интерес. Выписаны рекуррентные формулы из работы [6], которые позволяют найти распределения статистик (5) как в случае выполнения гипотезы $H_{0}$, так и при альтернативах $H_{q}$ к $H_{0}$. Эти формулы справедливы не только для конечных $N$, но и в случае счетного множества исходов испытаний (1). Ввиду равенства (6) указанные формулы позволяют также находить распределение статистики $\chi_{n}^{2}$. Указаны оценки сложности вычисления распределения статистики $\zeta_{n}^{(m)}$ при использовании рекуррентных формул, обсуждаемых в разделе 2.

Общий случай рассматривается в разделе 3 . Здесь получено представление для производящей функции произвольной статистики (4). С использованием этого представления описан алгоритм вычисления распределений разделимых статистик, который применим как при нулевой гипотезе, так и при альтернативах к ней. Показано, что этот алгоритм сводится к алгоритму А. М. Зубкова [7], описанному им в терминах некоторой двумерной цепи Маркова. Второй алгоритм, изложенный в разделе 3, применим лишь к симметричным разделимым статистикам в случае, когда справедлива гипотеза $H_{0}$. В его основе лежит одна рекуррентная формула Л. Эйлера, простое доказательство которой (одно из многих) можно найти в [8]. В заключение оценивается сложность вычисления распределения статистики $\chi_{n}^{2}$ с помощью алгоритмов, рассмотренных в разделе 3 .

\section{2. Одно частное множество разделимых статистик}

В этом разделе рассматривается множество статистик (5). Для $m=2,3, \ldots$ положим

$$
Q_{0,0}^{(m)}=1, \quad Q_{n, k}^{(m)}=\mathbf{P}\left\{\zeta_{N}^{(m)}=k\right\}, \quad n=2,3, \ldots, \quad k=0,1, \ldots,\left(\begin{array}{l}
n \\
m
\end{array}\right) .
$$

Эти обозначения относятся как к гипотезе $H_{0}$, так и к альтернативам $H_{q}$, введенным в разделе 1. Далее положим

$$
Q_{0}^{(m)}(y)=1, \quad Q_{n}^{(m)}(y)=\mathbf{E} y^{\zeta_{n}^{(m)}}=\sum_{k=0}^{\left(\begin{array}{c}
n \\
m
\end{array}\right)} Q_{n k}^{(m)} y^{k}, \quad n=1,2, \ldots
$$

Нам потребуются также многочлены

$$
d_{n}^{(m)}(y)=\sum_{k=0}^{\left(\begin{array}{c}
n \\
m
\end{array}\right)} d_{n, k}^{(m)} y^{k}, \quad n=1,2, \ldots
$$

не зависящие от $N$ и вероятностей $q_{1}, \ldots, q_{n}$, если справедлива альтернатива $H_{q}$. Многочлены (9) определяются равенствами

$$
d_{n}^{(m)}(y)=\sum_{k=0}^{\left(\begin{array}{c}
n \\
m
\end{array}\right)} c_{n k}^{(m)}(y-1)^{k}, \quad n=1,2, \ldots,
$$


где $c_{n k}^{(m)}, n=1,2, \ldots, k=0,1, \ldots,\left(\begin{array}{l}n \\ m\end{array}\right),-$ число связных гиперграфов с $n$ (помеченными) вершинами и $k$ ребрами, каждое из которых содержит $m$ вершин. В случае $m=2$ величина $c_{n k}^{(2)}$ есть число связных неориентированных графов без петель и параллельных ребер с $n$ помеченными вершинами и $k$ ребрами. Из (9) и (10) следует, что для коэффициентов $d_{n k}^{(m)}$ многочленов (9) выполняются равенства

$$
d_{n k}^{(m)}=\sum_{r=k}^{\left(\begin{array}{c}
n \\
m
\end{array}\right)}(-1)^{r-k}\left(\begin{array}{l}
r \\
k
\end{array}\right) c_{n k}^{(m)}, \quad n=1,2, \ldots, \quad k=0,1, \ldots,\left(\begin{array}{l}
n \\
m
\end{array}\right) .
$$

Мы можем сформулировать следующее предложение, доказанное в [6].

Теорема 1. Для многочленов (8) имеет место рекуррентное соотночение

$$
Q_{n+1}^{(m)}(y)=\sum_{i=0}^{n}\left(\begin{array}{l}
n \\
i
\end{array}\right) s_{i+1} d_{i+1}^{(m)}(y) Q_{n-i}^{(m)}(y), \quad n=0,1,2, \ldots,
$$

с начальным условием $Q_{0}^{(m)}(y)=1$. Величины $s_{k}$ в правой части (11) определяются равенствами

$$
s_{k}=\sum_{i=1}^{N} q_{i}^{k}, \quad k=1,2, \ldots
$$

и $s_{k}=N^{-(k-1)}$ в случае справедливости гипотезы $H_{0}$. Многочлены (9), входящие в правую часть (11), можно вычислять, используя соотношения

$$
d_{1}^{(m)}(y)=1, \quad d_{n+1}^{(m)}=y^{\left(\begin{array}{c}
n+1 \\
m
\end{array}\right)}-\sum_{k=1}^{n}\left(\begin{array}{l}
n \\
k
\end{array}\right) d_{n-k+1}^{(m)}(y) y^{\left(\begin{array}{c}
k \\
m
\end{array}\right)}, \quad n=1,2, \ldots
$$

С помощью теоремы 1 можно выписать отсутствующие в [6] рекуррентные соотношения непосредственно для коэффициентов многочленов (8) и (9). В частности, для $m=2$ справедливо следующее утверждение.

Следствие 1. Для $n=1,2, \ldots$ коэффициенты многочлена $d_{n}^{(2)}(y)$ удовлетворяют соотночениям

$$
d_{n+1, k}^{(2)}= \begin{cases}\sum_{m=1}^{n} d_{m, k-\left(\begin{array}{c}
n-m+1 \\
2
\end{array}\right),} & \text { если } k=0,1, \ldots,\left(\begin{array}{c}
n \\
2
\end{array}\right), \\
0, & \text { если } k=\left(\begin{array}{c}
n \\
2
\end{array}\right)+1, \ldots,\left(\begin{array}{c}
n+1 \\
2
\end{array}\right)-1, \\
1, & \text { если } k=\left(\begin{array}{c}
n+1 \\
2
\end{array}\right) .\end{cases}
$$

Здесь $d_{m k}^{(2)}=0$, если $k<0, d_{1,0}^{(2)}=1 u d_{1, k}^{(2)}=0$, если $k>0$.

Для коэффичиентов многочлена $Q_{n}^{(2)}(y)$ справедливы равенства

$$
\begin{aligned}
& Q_{n, k}^{(2)}=\sum_{i \in M(k)}\left(\begin{array}{c}
n-1 \\
i
\end{array}\right) s_{i+1} \sum_{j=0}^{\left(\begin{array}{c}
i+1 \\
2
\end{array}\right)} d_{i+1, j}^{(2)} Q_{n-i-1, k-j}^{(2)}, \quad k=0,1, \ldots,\left(\begin{array}{c}
n-1 \\
2
\end{array}\right), \\
& Q_{n, k}^{(2)}= \begin{cases}0, & k=\left(\begin{array}{c}
n-1 \\
2
\end{array}\right)+1, \ldots,\left(\begin{array}{c}
n \\
2
\end{array}\right)-1, \\
s_{n}, & k=\left(\begin{array}{c}
n \\
2
\end{array}\right),\end{cases}
\end{aligned}
$$


гдe

$$
\begin{aligned}
M(k) & =\{0,1, \ldots, n-1\}, \quad \text { если } k=0,1, \ldots,\left[\left((n-1)^{2}-1\right) / 4\right], \\
M(k) & =\left\{0,1, \ldots, m_{1}, m_{2}, \ldots, n-1\right\}, \text { если } k=\left[\left((n-1)^{2}-1\right) / 4\right]+1, \ldots,\left(\begin{array}{c}
n-1 \\
2
\end{array}\right)
\end{aligned}
$$

и уравнение

$$
m^{2}-(n-2) m+\left(\begin{array}{c}
n-1 \\
2
\end{array}\right)=k
$$

относительно $m$ имеет челочисленные решения $m_{1}, m_{2} \geqslant 0, m_{1}<m_{2}$, в противном случае $M(k)=\varnothing$. Здесь $[x]-$ челая часть $x$; если $M(k)=\varnothing$, то соответствуюшая сумма в (14) полагается равной нулю. Начальные условия в первом из равенств (14) имеют вид $Q_{0,0}^{(2)}=1, Q_{0, k}^{(2)}=0$, если $k>0$ или $k<0$, и $Q_{n k}^{(2)}=0$, если $n>0$, а $k<0$ или $k>\left(\begin{array}{c}n \\ 2\end{array}\right)$.

Вычисление распределений статистик $\zeta_{n}^{(m)}$ проще организовывать, используя соотношения (11) и (13), а не явные формулы, подобные тем, что указаны для $m=2$ в следствии 1. Из (11) видно, что для нахождения многочлена $Q_{n+1}^{(m)}(y), n \geqslant 1$, сначала нужно вычислить и записать в памяти компьютера многочлены $d_{1}^{(m)}(y), \ldots, d_{n+1}^{(m)}(y)$ и $Q_{1}^{(m)}(y), \ldots, Q_{n}^{(m)}(y)$. Для хранения коэффициентов всех многочленов требуется $O\left(n^{m+1}\right)$ ячеек памяти, а для их вычисления - $O\left(n^{2(m+1)}\right)$ машинных операций, поскольку многочлены $d_{n}^{(m)}(y)$ и $Q_{n}^{(m)}(y)$ имеют степень $\left(\begin{array}{l}n \\ m\end{array}\right)$. При этом объем требуемой памяти и число необходимых машинных операций не зависят от величины $N$, от которой зависят лишь суммы (12). В частности, для вычисления точного распределения статистики (7) с использованием рекуррентных соотношений теоремы 1 потребуется память объема $O\left(n^{3}\right)$ ячеек и $O\left(n^{6}\right)$ арифметических операций. Применяя результаты следствия 1 , за счет усложнения алгоритма можно уменьшить требования к памяти и трудоемкости.

В заключение этого раздела приведем некоторые сведения о предельных распределениях статистик $\zeta_{n}^{(m)}$. Если число исходов испытаний (1) фиксировано, а $n \rightarrow \infty$, то известно (см., например, замечание на с. 883 в [9]), что в случае выполнения гипотезы $H_{0}$ случайные величины $2(m-2) !(N / n)^{m-1}+N-2 n /(m(m-1)), m=2,3, \ldots$, сходятся по распределению к случайной величине хи-квадрат с $N-1$ степенями свободы. Если же выборка (1) получена при альтернативе $H_{q}$ и выполняется условие

$$
\sigma_{m}^{2}=s_{2 m-1}-s_{m}^{2}>0,
$$

где $s_{m}$ - соответствующая сумма (12), то согласно центральной предельной теореме для $U$-статистик ([10], с. 90) случайная величина $\sqrt{n}\left(\left(\begin{array}{l}n \\ m\end{array}\right)^{-1} \zeta_{n}^{(m)}-s_{m}\right)$ асимптотически нормальна с параметрами $\left(0, m^{2} \sigma_{m}^{2}\right)$. Можно показать, что условие (15) выполняется для всех $m=2,3, \ldots$, если альтернатива $H_{q}$ не принадлежит множеству альтернатив $H^{*}$, которое определено в [11] и строится следующим образом. Задаем различные подмножества $A \subset\{1,2, \ldots, N\}, 2 \leqslant|A|<N$, где $|A|-$ число элементов $A$; для $i=1, \ldots, N$ определяем вектор $q=\left(q_{1}, \ldots, q_{N}\right)$ с компонентами

$$
q_{i}= \begin{cases}0, & \text { если } i \in\{1, \ldots, N\} \backslash A, \\ |A|^{-1}, & \text { если } i \in A,\end{cases}
$$


и включаем в $H^{*}$ альтернативу $H_{q}$, соответствующую вектору $q$.

Наконец, предельные теоремы для статистик $\zeta_{n}^{(m)}$ при $n, N \rightarrow \infty$ можно извлечь из результатов Ю. И. Медведева [12].

\section{3. Общий случай}

Пусть $q_{1}, q_{2}, \ldots$ - произвольные комплексные числа. Рассмотрим формальные степенные ряды

$$
F_{i}(y, z)=\sum_{n=0}^{\infty} q_{i}^{n} y^{f_{i}(n)} z^{n} / n !, \quad i=1,2, \ldots
$$

и положим

$$
A_{k}(y, z)=\prod_{i=1}^{k} F_{i}(y, z)=\sum_{n=0}^{\infty} a_{n k}(y) z^{n} / n !, \quad k=1,2, \ldots
$$

Условимся, что сумма $\sum_{n_{1}+\ldots+n_{k}=n}$ распространяется на все возможные наборы целых неотрицательных чисел $n_{1}, \ldots, n_{k}$ таких, что $n_{1}+\ldots+n_{k}=n, k=1, \ldots, N$.

Нам потребуются следующие две леммы. Из (16) и (17) получаем следующее утверждение.

Лемма 1. Коэффичиенты рядов (17) определяются равенствами

$$
a_{n k}(y)=\sum_{n_{1}+\ldots+n_{k}=n} \frac{n !}{n_{1} ! \ldots n_{k} !} \prod_{i=1}^{k} q_{i}^{n_{i}} y^{\sum_{i=1}^{k} f_{i}\left(n_{i}\right)}
$$

где $k=1,2, \ldots, n=0,1, \ldots$, и удовлетворяют рекуррентному соотночению

$$
a_{n, k+1}(y)=\sum_{i=0}^{n}\left(\begin{array}{l}
n \\
i
\end{array}\right) q_{k+1}^{i} a_{n-i, k}(y) y^{f_{k+1}(i)}, \quad k=1,2, \ldots, \quad n=0,1, \ldots,
$$

с начальными условиями $a_{m 1}=q_{1}^{m} y^{f_{1}(m)}, m=0,1, \ldots$

Лемма 2. Пусть для испытаний (1) выполняется гипотеза $H_{q}$, компоненты вектора $q=\left(q_{1}, \ldots, q_{N}\right)$ удовлетворяют условиям (2) $u$

$$
g_{n, N}(y)=\sum_{c \in C(n, N)} \mathbf{P}\{L(n, N)=c\} y^{c}, \quad n=1,2, \ldots,
$$

где сумма $\sum_{c \in C(n, N)}$ берется по всем элементам с множества $C(n, N)$ различных значений статистики $L(n, N)$. Тогда в алгебре формальных степенных рядов над полем комплексных чисел справедливо равенство

$$
y^{L(0, N)}+\sum_{n=1}^{\infty} g_{n, N}(y) z^{n} / n !=\prod_{i=1}^{N} F_{i}(y, z) .
$$

Здесь $L(0, N)=\sum_{i=1}^{N} f_{i}(0) u F_{i}(y, z), i=1, \ldots, N,-p я \partial ы$ (16). 
Доказательство. Сумма (20) есть математическое ожидание случайной величины $y^{L(n, N)}$ (ее возможные значения - элементы множества $\left\{y^{c}: c \in C(n, N)\right\}$ ), то есть $g_{n, N}(y)=\mathbf{E} y^{L(n, N)}$. Поэтому $g_{n, N}(y)$ можно переписать в виде

$$
g_{n, N}(y)=\sum_{n_{1}+\ldots+n_{N}=n} \mathbf{P}\left\{v_{1}(n)=n_{1}, \ldots, v_{N}(n)=n_{N}\right\} y^{\sum_{i=1}^{N} f_{i}\left(n_{i}\right)} .
$$

Отсюда, используя известную формулу для полиномиальных вероятностей, получаем, что

$$
g_{n, N}(y)=\sum_{n_{1}+\ldots+n_{N}=n} \frac{n !}{n_{1} ! \ldots n_{N} !} \prod_{i=1}^{N} q_{i}^{n_{i}} y^{\sum_{i=1}^{N} f_{i}\left(n_{i}\right)}
$$

и, учитывая лемму 1, убеждаемся в справедливости леммы 2.

Следствие 2. Если

$$
L(n, N)=\sum_{i=1}^{N} f\left(v_{i}(n)\right)
$$

- симметричная разделимая статистика и выполняется гипотеза $H_{0}$, то

$$
y^{N f(0, N)}+\sum_{n=1}^{\infty} g_{n, N}(y) z^{n} / n !=\left(\sum_{n=0}^{\infty} y^{f(n)} z^{n} / n ! N^{n}\right)^{N} .
$$

где $g_{n, N}(y)$ определяется равенством (20).

Из равенства (21) легко выводится известное интегральное представление для характеристической функции разделимых статистик полиномиальной схемы (см. [13]). Частными случаями формулы (23) являются равенства (27) на с. 15 и (31) на с. 44 в [14], относящиеся к случайным величинам $\mu_{0}(n, N)$ и $\mu_{r}(n, N), r \geqslant 1$, в равновероятной схеме размещения. В неравновероятной схеме размещения частиц из формулы (21) следует одномерная версия теоремы 1 на с. 108 в [14]. Из (23) можно получить также представление для производящей функщии статистики $\sum_{i=1}^{N} v_{i}^{2}(n)$ в равновероятной полиномиальной схеме, указанное в [15]. Для статистик (5) из леммы 2 вытекает равенство

$$
1+\sum_{n=1}^{\infty} \sum_{k=0}^{\left(\begin{array}{c}
n \\
m
\end{array}\right)} Q_{n k}^{(m)} y^{k} z^{n} / n !=\prod_{i=1}^{N}\left(1+\sum_{n \geqslant 1} y^{\left.\left(\begin{array}{c}
n \\
m
\end{array}\right)\left(p_{i} z\right)^{n} / n !\right)},\right.
$$

которое представляет собой частный случай формулы (6) из работы [6], где она доказана для испытаний (1) со счетным множеством исходов.

Следствие 3. Предположим, что выполнены условия леммы 2. Тогда, используя рекурренту (19), можно последовательно для $k=1, \ldots, N-2$ вычислить функции $a_{m, k+1}(y)$, $m=0,1, \ldots, n$, затем для $k=N-1$ получить $a_{n, N}(y)=g_{n, N}(y) u$, таким образом, найти распределение разделимой статистики (4).

Следствие 3 описывает алгоритм вычисления распределения произвольной разделимой статистики полиномиальной схемы, который применим как в случае нулевой гипотезы, так и при альтернативах $H_{q}$ к $H$. Частный случай алгоритма, описанного в следствии 3 , указан в [16]. 
Точное распределение произвольной разделимой статистики полиномиальной схемы можно найти также с помощью рекуррентной формулы А. М. Зубкова (формула (10) в [7]): Чтобы выписать эту формулу, предположим, что выполняется гипотеза $H_{q}$, так что компоненты вектора $q=\left(q_{1}, \ldots, q_{N}\right)$ удовлетворяют условиям (2). Пусть, далее, $\pi_{1}, \ldots, \pi_{N}-$ независимые пуассоновские случайные величины и $\pi_{k}$ имеет параметр $\lambda q_{k}, k=1, \ldots, N$ ( $\lambda$ - положительная постоянная), так что

$$
\mathbf{P}\left\{\pi_{k}=n\right\}=\frac{\left(\lambda q_{k}\right)^{n}}{n !} e^{-\lambda q_{k}}, \quad k=1, \ldots, N, \quad n=0,1, \ldots
$$

Тогда для частот исходов в $n$ испытаниях (1) имеет место известная формула

$$
\mathbf{P}\left\{\nu_{k}(n)=n_{k}, k=1, \ldots, N\right\}=\mathbf{P}\left\{\pi_{k}=n_{k}, k=1, \ldots, N \mid \pi_{1}+\ldots+\pi_{N}=n\right\} .
$$

С помощью случайных величин $\pi_{1}, \ldots, \pi_{N}$ и функций $f_{1}(x), \ldots, f_{N}(x)$, задающих разделимую статистику (4), в [7] определена двумерная цепь Маркова $\left(L_{k}, \Pi_{k}\right)$, $k=0,1, \ldots, N$, где

$$
L_{0}=\Pi_{0}=0, \quad L_{k}=\sum_{i=1}^{k} f_{i}\left(\pi_{i}\right), \quad \Pi_{k}=\sum_{i=1}^{k} \pi_{k}, \quad k=1, \ldots, N,
$$

и показано, что

$$
\mathbf{P}\left\{L_{k+1}=c, \Pi_{k+1}=n\right\}=\sum_{m=o}^{n} \mathbf{P}\left\{L_{k}=c-f_{k+1}(m), \Pi_{k}=n-m\right\} \mathbf{P}\left\{\pi_{k+1}=m\right\},
$$

где $c \in C_{k+1}$ и $C_{1}, \ldots, C_{N}-$ множества возможных значений случайных величин $L_{1}, \ldots, L_{N}$, соответственно. Формула (25) определяет рекуррентную процедуру, которая позволяет найти распределение случайной величины $L_{N}$, а затем с учетом (24) и статистики $L(n, N)$.

Теорема 2. Если $q_{1}, \ldots, q_{N}$ - действительные числа, удовлетворяющие условию (2), то соотночение (19) выполняется тогда и только тогда, когда имеет место равенство (25).

Доказательство. Покажем, что из (19) следует (25). Воспользуемся формулой (18) и представим (19) в виде

$$
\begin{aligned}
\sum_{n_{1}+\ldots+n_{k+1}=n}\left(\prod_{i=1}^{k+1} q_{i}^{n_{i}} / n_{i} !\right) y^{\sum_{i=1}^{k+1} f_{i}\left(n_{i}\right)} & \\
& =\sum_{m=0}^{n}\left(q_{k+1}^{m} / m !\right) y^{f_{k+1}(m)} \sum_{n_{1}+\ldots+n_{k}=n-m}\left(q_{i}^{n_{i}} / n_{i} !\right) y^{\sum_{i=1}^{k} f_{i}\left(n_{i}\right)},
\end{aligned}
$$

где $k=1, \ldots, N-1, n=0,1, \ldots$ Умножим обе части равенства на $\lambda^{n} e^{\lambda\left(q_{1}+\ldots+q_{k+1}\right)}$ и, учитывая независимость случайных величин $\pi_{1}, \ldots, \pi_{k+1}$, получим, что

$$
\begin{aligned}
& \sum_{n_{1}+\ldots+n_{k+1}=n} \mathbf{P}\left\{\pi_{1}=n_{1}, \ldots, \pi_{k+1}=n_{k+1}\right\} y^{\sum_{i=1}^{k} f_{i}\left(n_{i}\right)} \\
= & \sum_{m=0}^{n} \mathbf{P}\left\{\pi_{k+1}=m\right\} y^{f_{k+1}(k)} \sum_{n_{1}+\ldots+n_{k}=n-m} \mathbf{P}\left\{\pi_{1}=n_{1}, \ldots, \pi_{k}=n_{k}\right\} y^{\sum_{i=1}^{k} f_{i}\left(n_{i}\right)},
\end{aligned}
$$


где $k=1, \ldots, N-1, n=0,1, \ldots$ Заметим, что для $l=k, k+1$

$$
\sum_{n_{1}+\ldots+n_{l}=n} \mathbf{P}\left\{\pi_{1}=n_{1}, \ldots, \pi_{l}=n_{l}\right\} y^{\sum_{i=1}^{k} f_{i}\left(n_{i}\right)}=\sum_{c \in C_{l}} \mathbf{P}\left\{L_{l}=c, \Pi_{l}=n\right\} y^{c} .
$$

Поэтому (26) приводится к виду

$$
\begin{aligned}
& \sum_{c \in C_{l+1}} \mathbf{P}\left\{L_{k+1}=c, \Pi_{k+1}=n\right\} y^{c} \\
&=\sum_{c \in C_{k}} \sum_{m=0}^{n} \mathbf{P}\left\{L_{k}=c^{\prime}, \Pi_{k}=n-m\right\} \mathbf{P}\left\{\pi_{k+1}=m\right\} y^{c^{\prime}+f_{k+1}(m)},
\end{aligned}
$$

откуда получаем (25).

Ясно, что, повторяя предыдущие рассуждения в обратном порядке, из (25) получим (19). Теорема доказана.

В ходе доказательства теоремы 2 фактически были получены равенства

$$
\frac{\lambda^{n}}{n !} e^{-\lambda\left(q_{1}+\ldots+q_{k}\right)} a_{n, k, c}=\mathbf{P}\left\{L_{k}=c, \Pi_{k}=n\right\}, \quad c \in C_{k}, \quad k=1, \ldots, N,
$$

в которых величины $a_{n, k, c}$ определяются из разложения

$$
a_{n, k}(y)=\sum_{c \in C_{k}} a_{n, k, c} y^{c}
$$

а другие величины были определены выше в разделе 3.

Сложность вычисления допредельного распределения статистики $L(n, N)$ линейно зависит от $N$ и более сложным образом от $n$. В общем случае эта сложность зависит от мощности множества $C_{N}$ ее различных значений. Ряд замечаний по этому вопросу можно найти в [7].

Если разделимая статистика, распределение которой требуется вычислить, является симметричной и в испытаниях (1) реализуется равновероятная полиномиальная схема, можно предложить менее трудоемкий алгоритм.

Теорема 3. Если верна гипотеза $H_{0}$, то для многочленов (20), определяющих распределение симметричной разделимой статистики (22), выполняется рекуррентное соотношение

$$
q_{n, N}(y)=\sum_{k=1}^{n} \frac{1}{N^{k}}\left(N+1-\frac{n}{k}\right)\left(\begin{array}{l}
n-1 \\
k-1
\end{array}\right) y^{f(k)-f(0)} g_{n-k, N}(y), \quad n=1,2, \ldots
$$

с начальным условием $g_{0, N}=y^{N f(0)}$.

Доказательство. Применим к коэффициентам ряда из левой части равенства (23) соотношение (2.16) из [8]. Положим в последнем соотношении $r=N$ и при $n=1,2, \ldots$, $x_{n}=\left(1 / N^{n}\right) y^{f(n)}, X_{n}^{(N)}=g_{n, N}(y)$, получим формулу (27).

Используя теорему 2.2 и следствие 2.1 из [8], в равновероятной полиномиальной схеме для производящих функций $g_{n, N}(y)$ симметричных разделимых статистик можно выписать ряд других рекуррентных формул и тождеств. 
Оценим сложность нахождения распределения статистики $\chi_{n}^{2}$, заданной формулой (7), с использованием рекуррентных формул (19) и (27). При этом удобно использовать целочисленную случайную величину $v^{2}(n)=\sum_{i=1}^{N} v_{i}^{2}(n)$, так как с учетом (7) находим, что $\mathbf{P}\left\{\chi_{n}^{2}=N k / n-n\right\}=\mathbf{P}\left\{v^{2}(n)=k\right\}, k=0,1, \ldots, n^{2}$, и $\mathbf{P}\left\{v^{2}(n)=k\right\}=0$ для значений $k$, отличных от $0,1, \ldots, n^{2}$. Согласно следствию 3 при вычислении распределения величины $v^{2}(n)$ с использованием рекурренты (19) нужно иметь память объема $O\left(n^{3}\right)$ ячеек для хранения коэффициентов многочленов $a_{k, m}(y), a_{k+1, m}(y), m=1, \ldots, n$, и потребуется произвести $O\left(\mathrm{Nn}^{4}\right)$ арифметических операций. В случае использования формулы (27), когда верна гипотеза $H_{0}$, для вычисления распределения $v^{2}(n)$ необходимый объем памяти также есть $O\left(n^{3}\right)$, но число арифметических операций есть $O\left(n^{4}\right)$.

\section{Список литературы}

1. Крамер Г., Математические методы статистики. ИЛ, Москва, 1948.

2. Ван дер Варден Б. Л., Математическая статистика. ИЛ, Москва, 1960.

3. Ивченко Г. И., Медведев Ю. И., Математическая статистика. Высшая школа, Москва, 1984.

4. Good I. J., Gover T. N., Mitchell G. J., Exact distributions for $\chi^{2}$ and for likelihood-ratio statistic for the equiprobable multinomial distribution. J. Amer. Statist. Assoc. (1970) 65, 267-283.

5. Holzman G. I., Good I. J., The Poisson and chi-squared approximation as compared with the true upper-tail probabillity of Pearson's $\chi^{2}$ for equiprobable miltinomials. J. Statist. Plann. Inference (1986) 13, №3, 283-295.

6. Селиванов Б. И., О производящей функции одной статистики в полиномиальной схеме со счетным множеством исходов. Комбинаторный анализ (1972) 2, 11-16.

7. Зубков А. М., Рекуррентные формулы для распределений функционалов от дискретных случайных величин. Обозрение прикладной и промышленной математики (1996) 3, №4, 567-573.

8. Селиванов Б. И., О комбинаторных функциях, связанных с рядом Бюрмана-Лагранжа. Соотношения квазиортогональности. Теория вероятностей и ее применения (1998) 10, №1, 127-140.

9. Михайлов В. Г., Центральная предельная теорема для числа неполных длинных повторений. Теория вероятностей и ее применения (1975) 20, №4, 880-884.

10. Королюк В. С., Боровских Ю. В., Теория $U$-статистик. Наукова Думка, Киев, 1989.

11. Селиванов Б. И., О предельных распределениях статистики $\chi^{2}$. Теория вероятностей и ее применения (1984) 29, №1, 132-134.

12. Медведев Ю. И., Разделимые статистики в полиномиальной схеме, I, II. Теория вероятностей и ее применение (1977) 22, №1, 3-17; №3, 623-631.

13. Медведев Ю. И., Некоторые теоремы об асимптотическом распределении статистики $\chi^{2}$. Доклады АН СССР (1970) 192, №5, 987-980.

14. Колчин В. Ф., Севастьянов Б. А., Чистяков В. П., Случайные размещения. Наука, Москва, 1976.

15. Good I. J., Saddle-point methods for the multinomial distribution. Ann. Math. Statist. (1957) 28, №4, 861-881.

16. Селиванов Б. И., О времени ожидания в схеме случайных размещений окрашенных частиц. Дискретная математика (1995) 7, №1, 134-144.

Статья поступила 09.11.2004. Переработанный вариант поступил 12.12.2005. 